\title{
DETERMINAÇÃO DA COMPOSIÇÃO ISOTÓPICA DE ESTRÔNCIO EM ÁGUAS NATURAIS: EXEMPLOS DE SUA APLICAÇÃ̃o EM ÁGUAS SUBSUPERFICIAIS DA ZONA COSTEIRA NA REGIÃO BRAGANTINA-PA
}

\author{
Adriana Oliveira Bordalo*, Candido Augusto Veloso Moura e Thomas Scheller \\ Centro de Geociências, Universidade Federal do Pará, CP 1611, 66075-900 Belém - PA, Brasil
}

Recebido em 20/3/06; aceito em 6/10/06; publicado na web em 28/5/07

\begin{abstract}
DETERMINATION OF STRONTIUM ISOTOPIC COMPOSITION IN NATURAL WATERS: EXAMPLES OF APPLICATION IN SUBSURFACE WATERS OF THE COASTAL ZONE OF BRAGANTINA REGION, PARÁ. Analytical procedures used for determining the concentrations and isotope composition of strontium in subsurface waters, by mass spectrometry, are described. Sampling was performed in coastal plateaus, salt marsh and mangrove environments in the coastal region of Pará. Coastal plateau waters have $\delta^{87} \mathrm{Sr}$ between 1.51 and $6.26 \%$ and $\mathrm{Sr}$ concentration bellow $58 \mathrm{ppb}$. Salt marsh waters show $\delta^{87} \mathrm{Sr}$ between 0.55 and $0.90 \%$ and $\mathrm{Sr}$ concentration between 93 and $114 \mathrm{ppm}$, while mangrove waters have $\delta^{87} \mathrm{Sr} \%$ oround zero and $\mathrm{Sr}$ concentration above $15 \mathrm{ppm}$. Differences in the ${ }^{87} \mathrm{Sr} /{ }^{86} \mathrm{Sr}$ ratio in these subsurface waters are detected, as well as seasonal variations in the coastal pleteau waters.
\end{abstract}

Keyword: Sr concentration; Sr isotopes; subsurface water.

\section{INTRODUÇÃO}

A razão isotópica ${ }^{87} \mathrm{Sr} /{ }^{86} \mathrm{Sr}$ tem sido empregada por um número crescente de pesquisadores na determinação de fontes de contaminação e misturas de águas naturais ${ }^{1-6}$. Os isótopos de $\mathrm{Sr}$ vêm sendo utilizados também na investigação dos efeitos da agricultura nas águas subterrâneas e superficiais? ${ }^{7}$. Adicionalmente, são usados na determinação do grau de interação rocha-água e identificação de águas superficiais e subterrâneas afetadas por drenagem ácidas de minas ${ }^{8,9}$.

Em função da crescente utilização desse método no estudo de águas naturais e a contribuição em potencial dessa ferramenta para o estudo dos recursos hídricos, este trabalho relata a implantação da análise isotópica de $\mathrm{Sr}$ em águas naturais no Laboratório de Geologia Isotópica da Universidade Federal do Pará (Pará-Iso). Este laboratório realiza, rotineiramente, análises isotópicas de $\mathrm{Sr}$ em minerais e rochas e algumas adaptações metodológicas foram implementadas para análise de águas naturais. Na implantação do método dois procedimentos merecem ser destacados. O primeiro foi o emprego de resina específica de $\mathrm{Sr}$ (Sr.spec) para a separação cromatográfica de Sr. Para tanto adaptaram-se os procedimentos analíticos experimentais desenvolvidos por Horwitz et al. ${ }^{10}$. O outro ponto importante foi o procedimento adotado na tentativa de baixar o nível de contaminação de Sr no laboratório, visto que a concentração de $\mathrm{Sr}$ em águas naturais é normalmente muito baixa. O pequeno volume de reagentes utilizados na separação cromatográfica e os cuidados na preparação desses reagentes permitiram uma diminuição considerável no nível de contaminação laboratorial.

$\mathrm{Na}$ implantação da análise isotópica de $\mathrm{Sr}$ em águas naturais, procurou-se testar inicialmente a aplicação desta ferramenta em águas subsuperficiais. Para isso, foi selecionada a região costeira do nordeste do Pará, onde estudos geológicos têm revelado a presença de diversos paleoambientes, distintamente afetados pela água do marr $^{11}$. Com base nessas informações selecionaram-se 3 sítios de amostragem de águas subsuperficiais. Um situado em rochas silicáticas terciárias, onde as águas de subsuperfície deveriam registrar uma assinatura isotópica continental. O outro sítio localizase em uma zona transicional em ambiente de pântano salino. O

*e-mail: adriana@ufpa.br terceiro sítio foi selecionado em uma zona de mangue intermaré, portanto, com forte influência marinha.

\section{GEOLOGIA ISOTÓPICA DO ESTRÔNCIO (Sr) E SUA UTILIZAÇÃO EM ÁGUAS NATURAIS}

O estrôncio ( $\mathrm{Sr}$ ) é um elemento químico do grupo dos metais alcalinos terrosos, que substitui o $\mathrm{Ca}$, de forma restrita, em minerais como plagioclásio, apatita e carbonato de cálcio ${ }^{12}$. O Sr está presente em águas naturais em quantidades variáveis. Nos oceanos apresenta uma concentração média de $7,7 \mathrm{mg} / \mathrm{L}^{12}$. Nos rios da Amazônia a concentração de Sr no material em suspensão varia de 40 a 176 $\mathrm{mg} / \mathrm{L}$, e em solução situa-se entre 4,3 e $39 \mu \mathrm{g} / \mathrm{L}^{3,13}$. A concentração de $\mathrm{Sr}$ em águas subterrâneas varia de 6 a $980 \mu \mathrm{g} / \mathrm{L}^{4,14}$ e a concentração relatada em águas pluviais varia de 96 a $874 \mu \mathrm{g} / \mathrm{L}^{15}$.

$\mathrm{O}$ estrôncio tem quatro isótopos estáveis que ocorrem naturalmente, ${ }^{84} \mathrm{Sr},{ }^{86} \mathrm{Sr},{ }^{87} \mathrm{Sr},{ }^{88} \mathrm{Sr}$. O isótopo mais abundante é o ${ }^{88} \mathrm{Sr}$ com $82,53 \%$, e o menos abundante é o ${ }^{84} \mathrm{Sr}$, que perfaz apenas $0,56 \%$ do total dos átomos de $\mathrm{Sr} . \mathrm{O}{ }^{87} \mathrm{Sr}$ é radiogênico, proveniente do decaimento radioativo do ${ }^{87} \mathrm{Rb}$ pela emissão de uma partícula $\beta$. Em função disso, a razão ${ }^{87} \mathrm{Sr} /{ }^{86} \mathrm{Sr}$ varia com o tempo e em função da razão $\mathrm{Rb} / \mathrm{Sr}$ do material. Os valores da razão ${ }^{87} \mathrm{Sr} /{ }^{86} \mathrm{Sr}$ do $\mathrm{Sr}$ dissolvido na água do mar situam-se na faixa de 0,709177 e $0,70923^{16}$. Essa razão é considerada homogênea devido ao longo tempo de residência do $\mathrm{Sr}$ nos oceanos, da ordem de $5 \times 10^{6}$ anos, quando comparado com o tempo de mistura das águas oceânicas, de cerca de $10^{3} \operatorname{anos}^{17}$. No entanto, pequenas variações na razão ${ }^{87} \mathrm{Sr} /{ }^{86} \mathrm{Sr}$ são observadas em regiões estuarinas onde ocorrem "inputs" de material continental ${ }^{13}$.

A composição isotópica do Sr nas águas naturais é variável e depende da idade e da razão $\mathrm{Rb} / \mathrm{Sr}$ das rochas que essas águas percolam ou escoam, bem como da solubilidade relativa dos diferentes minerais que podem estar em contato com essas águas. A razão ${ }^{87} \mathrm{Sr} r{ }^{86} \mathrm{Sr}$ no material em suspensão dos rios da Amazônia varia entre 0,71319 e 0,75640 , enquanto que no material dissolvido esta razão é mais baixa e varia de 0,708776 a $0,733172^{3}$. Isso pode ser explicado pelo fato do material dissolvido conter uma parte significativa de $\mathrm{Sr}$ derivado de águas pluviais, que possuem razão ${ }^{87} \mathrm{Sr}^{86} \mathrm{Sr}^{3}$ mais baixa. A composição isotópica do $\mathrm{Sr}$ em águas pluviais situa-se entre aquela da água do 
mar e a de rochas carbonáticas ${ }^{3}$, cujo valor, no Fanerozóico, tem sido superior a $0,707^{18}$. Conseqüentemente, os valores da razão ${ }^{87} \mathrm{Sr} /{ }^{86} \mathrm{Sr}$ das águas pluviais situam-se, aproximadamente, entre 0,707 e 0,709. Valores entre 0,70792 e 0,70917 foram obtidos para a razão ${ }^{87} \mathrm{Sr} /{ }^{86} \mathrm{Sr}$ de águas pluviais em Israel ${ }^{15}$.

Banner ${ }^{13}$ ilustrou a relação entre a razão ${ }^{87} \mathrm{Sr} /{ }^{86} \mathrm{Sr}$ das águas superficiais e os diferentes terrenos que elas drenam. Segundo este autor, a razão ${ }^{87} \mathrm{Sr} /{ }^{86} \mathrm{Sr}$ em torno de 0,710 , para alguns dos maiores rios que drenam o sul da América do Norte, seria consistente com a combinação de Sr proveniente de crosta do Proterozóico e Paleozóico. Por sua vez, o valor médio da razão ${ }^{87} \mathrm{Sr} /{ }^{86} \mathrm{Sr}$ de 0,711 dos rios canadenses refletiria a contribuição de crosta mais antiga do escudo Pré-cambriano, enquanto valores mais baixos de ${ }^{87} \mathrm{Sr} /{ }^{86} \mathrm{Sr}(0,704-0,708)$ são encontrados em rios que drenam terrenos vulcânicos do Pacífico, como Japão e Filipinas, que transportam $\mathrm{Sr}$ proveniente do intemperismo de rochas derivadas do manto em épocas mais recentes.

A variação da razão isotópica de $\mathrm{Sr}$ em águas subterrâneas é similar àquela de águas superficiais. A razão ${ }^{87} \mathrm{Sr} /{ }^{86} \mathrm{Sr}$ é uma mistura da composição isotópica do Sr da água de recarga com a composição isotópica da rocha que a água percola. No entanto, esta variação é bem mais pronunciada em águas subterrâneas, em conseqüência de mais altas temperatura, pressão e força iônica dessas águas, bem como do longo tempo de percolação e da alta razão entre a superfície mineral e o volume de água ${ }^{13}$. Nas águas subterrâneas da Austrália, Collerson et al. ${ }^{19}$ encontraram valores da razão ${ }^{87} \mathrm{Sr} /{ }^{86} \mathrm{Sr}$ variando entre 0,70446 e 0,71176 . Os valores mais baixos foram interpretados como a mistura entre a água de recarga do aqüífero australiano com rochas ígneas máficas cenozóicas, que possuem uma assinatura isotópica ${ }^{87} \mathrm{Sr} /{ }^{86} \mathrm{Sr}$ juvenil.

A variação na composição isotópica do $\mathrm{Sr}$ tem sido usada para determinar a fonte de $\mathrm{Sr}$ de um corpo de água em particular, a história geoquímica dessas águas (interação rocha-água) e o potencial de mistura dessas águas ${ }^{2}$. Registros dessas aplicações podem ser encontrada no trabalho de Palmer e Edmond ${ }^{1}$, que estudaram a composição isotópica do $\mathrm{Sr}$ em diferentes bacias de drenagem, para investigar os mecanismos que resultam no aumento da razão ${ }^{87} \mathrm{Sr} /{ }^{86} \mathrm{Sr}$ nas águas dos rios. Naftz et al. ${ }^{20}$ usaram valores de $\delta^{87} \mathrm{Sr}$ para identificar a fonte salina em um aqüífero de água doce no estado de Utah, nos Estados Unidos. Lyons et al. ${ }^{2}$ usaram os isótopos de $\mathrm{Sr}$ para determinar a mistura de água subterrânea com água salobra em uma zona costeira na Austrália.

\section{IMPLANTAÇÃO DO MÉTODO DE ANÁLISE ISOTÓPICA DE Sr EM ÁGUAS NATURAIS}

\section{Preparação e purificação de reagentes}

Os reagentes químicos contribuem bastante para a contaminação das amostras, o que obriga a purificação dos mesmos. Dessa forma, os reagentes utilizados $\left(\mathrm{HCl}, \mathrm{HNO}_{3}\right.$ e $\left.\mathrm{H}_{2} \mathrm{O}\right)$ foram purificados por destilação em destilador de quartzo.

A água utilizada para a preparação dos reagentes empregados neste trabalho foi destilada 4 vezes $\left(\mathrm{H}_{2} \mathrm{O} * * * *\right)$, enquanto a água para a limpeza do material empregado foi 3 vezes destilada $\left(\mathrm{H}_{2} \mathrm{O}^{* * *}\right)$.

As soluções ácidas destinadas à análise foram preparadas a partir de reagentes 2 vezes destilados $\left(\mathrm{HCl}^{* *}\right.$ e $\left.\mathrm{HNO}_{3} * *\right)$ e diluídos em $\mathrm{H}_{2} \mathrm{O} * * * *$.

\section{Separação cromatográfica do $\mathrm{Sr}$}

Para determinação da composição isotópica de um dado elemento químico, por espectrometria de massas com fonte de ionização termal (TIMS), o elemento deve ser rigorosamente sepa- rado dos demais, para que não ocorram interferências isobáricas e dificuldades de ionização, que resultam em análises de baixa confiabilidade. A extração cromatográfica tem sido uma importante ferramenta para isolar o elemento de interesse.

$\mathrm{O} \mathrm{Ca}$ e o $\mathrm{Rb}$ são elementos interferentes e precisam ser totalmente separados do Sr para a análise isotópica de Sr por TIMS. O $\mathrm{Ca}$, por ter grande similaridade química com o $\mathrm{Sr}$, é de difícil separação cromatográfica. A presença de Ca residual diminui a eficiência de ionização do $\mathrm{Sr}$ e a estabilidade do feixe de íons durante a análise por TIMS. Por sua vez, o perfeito isolamento de $\mathrm{Sr}$ do $\mathrm{Rb}$ é necessário para a determinação precisa da razão ${ }^{87} \mathrm{Sr} /{ }^{86} \mathrm{Sr}$, devido à interferência isobárica do ${ }^{87} \mathrm{Rb}$.

$\mathrm{Na}$ separação cromatográfica do $\mathrm{Sr}$, utilizou-se a resina específica de $\mathrm{Sr}$, fabricada pela indústria Eichrom com nome comercial sr.Spec. Esta resina consiste de uma solução de éter de coroa DtBuCH18-C6 (18-crown-6) em 1 octanol, suportada pelo substrato inerte Amberchrom CG-72ms ${ }^{21}$. A resina Sr.spec, cuja capacidade é de $8,9 \mathrm{mg} \mathrm{Sr} / \mathrm{mL}$ de resina, combina a força e a seletividade da extração por solvente facilitando o uso desta resina cromatográfica. A utilização de pequenas colunas na eluição do Sr implica na redução do volume de reagentes, diminuindo o custo e o tempo de análise. Além disso, a resina Sr.spec possibilita uma excelente separação do $\mathrm{Ca}$ e $\mathrm{Rb}$ do $\mathrm{Sr}$, com recuperação de até $95 \%$ do $\mathrm{Sr}^{22,23}$.

A resina específica de $\mathrm{Sr}$ com granulometria entre 50 e 100 $\mathrm{mm}$, empregada neste método, é livre de interferências de muitos metais, como $\mathrm{Ca}, \mathrm{Al}$ e $\mathrm{Fe}$, mesmo que esses metais estejam presentes em quantidades que excedam a capacidade da resina. Essa propriedade faz com que a resina Sr.spec seja ideal para separação de Sr envolvendo amostras ambientais ${ }^{10}$.

Antes da utilização da resina Sr.spec, a mesma foi lavada 5 vezes em $\mathrm{H}_{2} \mathrm{O}^{* * * * *}$ por agitação a quente $\left(50^{\circ} \mathrm{C}\right)$. Após a lavagem, a resina foi acondicionada em $\mathrm{H}_{2} \mathrm{O} * * * *$ à $15^{\circ} \mathrm{C}$.

As colunas de separação foram confeccionadas em Teflon ${ }^{\circledR}$ termo-retrátil com 10,4 mm de diâmetro e possuem um disco de Teflon ${ }^{\circledR}$ (3,4 mm de diâmetro), utilizado para retenção da resina durante a separação cromatográfica. Na preparação inicial das colunas, com auxílio de um cilindro de vidro, o Teflon ${ }^{\circledR}$ foi moldado com ar quente em uma das extremidades para possibilitar a introdução do disco de Teflon ${ }^{\circledR}$ (Figura 1).

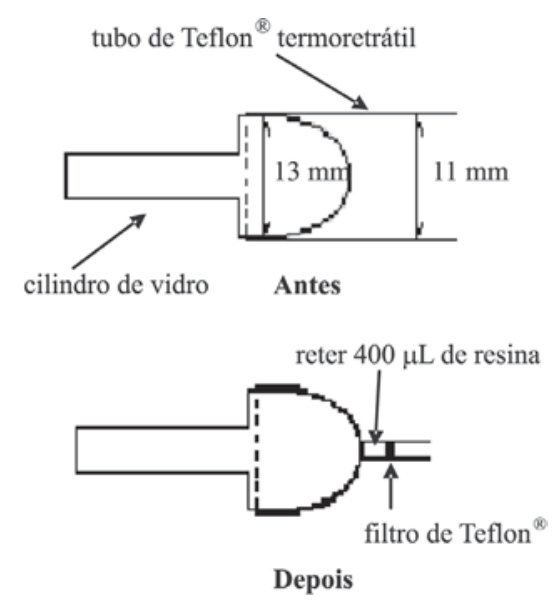

Figura 1. Ilustração da confecção das colunas de téflon para separação de $\mathrm{Sr}$

A separação cromatográfica do $\mathrm{Sr}$ inicia-se com a introdução de $\mathrm{H}_{2} \mathrm{O} * * * *$ seguida da adição de $250 \mu \mathrm{L}$ de resina. A limpeza da resina é efetuada com duas lavagens sucessivas de $500 \mu \mathrm{L}$ de $\mathrm{H}_{2} \mathrm{O}^{* * * *}$, e o condicionamento com $500 \mu \mathrm{L}$ de $\mathrm{HNO}_{3} * * 3,5 \mathrm{~mol} /$ L. Em seguida, adiciona-se $500 \mu \mathrm{L}$ da amostra solubilizada em 
$\mathrm{HNO}_{3}^{* *} 3,5 \mathrm{~mol} / \mathrm{L}$. Realiza-se o enxágüe da resina adicionando 1200 $\mu \mathrm{L}$ de $\mathrm{HNO}_{3}^{* *} 3,5 \mathrm{~mol} / \mathrm{L}$ para a retirada dos outros elementos químicos presentes na amostra, ficando o $\mathrm{Sr}$ retido na resina. A separação do $\mathrm{Sr}$, propriamente dita, ocorre com a adição de $1000 \mu \mathrm{L}$ de $\mathrm{H}_{2} \mathrm{O} * * * *$. O Sr eluído é recuperado em béquer de Teflon ${ }^{\circledR}$ onde adiciona-se $10 \mu \mathrm{L}$ de $\mathrm{H}_{3} \mathrm{PO}_{4} 0,1 \mathrm{M}$ para aglutinar o Sr presente na amostra. Finalmente, o concentrado de $\mathrm{Sr}$ é evaporado a $90{ }^{\circ} \mathrm{C}$.

A resina é descartada, a coluna enxaguada com $\mathrm{H}_{2} \mathrm{O}^{* * *}$ e estocada em $\mathrm{HNO}_{3} * * 7 \mathrm{~mol} / \mathrm{L}$.

\section{Análise isotópica do $\mathrm{Sr}$ por espectrometria de massas}

$\mathrm{O}$ espectrômetro de massas empregado foi o VG $54 \mathrm{E}$, equipado com um setor magnético $\left(90^{\circ}\right)$, uma fonte de ionização termal e um sistema de monocoletor de íons composto por um copo de Faraday e um detector Daly.

Para a análise do Sr por espectrometria de massas, foi utilizado filamento simples de $\mathrm{W}$. Adiciona-se ao filamento $1 \mu \mathrm{L}$ de solução ativadora de Ta e, em seguida, deposita-se $1 \mu \mathrm{L}$ da solução que contém o concentrado de $\mathrm{Sr}$ previamente dissolvido com $\mathrm{HNO}_{3} * *$ ( $\sim 6 \mathrm{~mol} / \mathrm{L})$. As análises isotópicas foram realizadas no período de 8 meses, onde se procedeu ao controle da precisão analítica do equipamento realizando 7 medidas do material de referência SRM 987, que forneceram uma média $0,710219 \pm 0,000014$ para a razão ${ }^{87} \mathrm{Sr} /{ }^{86} \mathrm{Sr}$. O valor fornecido no certificado NIST para SRM $987 \mathrm{da}$ razão ${ }^{87} \mathrm{Sr} /{ }^{86} \mathrm{Sr}$ é de $0,71034 \pm 0,00026$.

\section{Diluição isotópica e preparação da solução traçadora}

O espectrômetro de massas pode ser usado para determinar a concentração do elemento em uma amostra através da diluição isotópica ${ }^{24,25}$. Esse método é baseado na determinação da composição isotópica de um elemento, através da mistura de uma quantidade conhecida de traçador com uma quantidade desconhecida de um elemento. $\mathrm{O}$ traçador é uma solução (líquida ou gasosa) que contém uma concentração conhecida de um elemento particular no qual a composição isotópica é alterada pelo enriquecimento de um dos isótopos.

A amostra a ser analisada contém uma concentração desconhecida de um elemento cuja composição isotópica é conhecida. Quando uma determinada quantidade de amostra é misturada com uma determinada quantidade de traçador, a composição isotópica da mistura pode ser usada para calcular a quantidade do elemento na amostra. Um traçador específico de $\mathrm{Sr}$ para realização da análise isotópica de $\mathrm{Sr}$ em águas naturais foi preparado.

A solução traçadora mãe foi preparada a partir da diluição em $\mathrm{HCl} 2 \mathrm{~mol} / \mathrm{L}$ do traçador sólido $\mathrm{Sr}\left(\mathrm{NO}_{3}\right)_{2}$, enriquecido em ${ }^{84} \mathrm{Sr}(81$ $\%)$. Cerca de $100 \mu \mathrm{L}$ desta solução foi misturada com $200 \mu \mathrm{L}$ de Titrisol $^{\circledR}$ (concentrado de Sr). Este procedimento foi realizado para determinação por diluição isotópica da concentração de ${ }^{84} \mathrm{Sr}$ na solução mãe.

Após esta etapa, $100 \mu \mathrm{L}$ da solução mãe foram diluídos com $100 \mathrm{~mL}$ de $\mathrm{HCl} 2,5 \mathrm{~mol} / \mathrm{L}$, para fabricação do traçador de $\mathrm{Sr}$ utilizado. As razões isotópicas de $\mathrm{Sr}$ deste traçador são: 86/84 = 0,$054998 ; 87 / 84=0,021387 ; 88 / 84=0,165433$.

A concentração de ${ }^{84} \mathrm{Sr}$ no traçador de $\mathrm{Sr}$ foi determinada misturando-o com solução Titrisol ${ }^{\circledR}$. A mistura é evaporada e a composição isotópica determinada por espectrometria de massas.

\section{Controle de contaminação laboratorial}

Análises dos brancos dos procedimentos são realizadas com a finalidade de estimar a contaminação introduzida durante o procedimento químico. Esse controle pode ser feito repetindo-se o pro- cedimento usual sem a presença da amostra e em conjunto com o lote de amostras permitindo, assim, determinar a quantidade de $\mathrm{Sr}$ introduzido como contaminante.

O controle do branco total de Sr no Pará-Iso realizado no período de 1996 a 1999 mostrou teores decrescentes, variando de 9 a 4 ng de Sr. Com o procedimento adotado neste trabalho alcançou-se um branco total de $\mathrm{Sr}$ de $2 \mathrm{ng}$ e, atualmente, este branco é inferior a $1 \mathrm{ng}$. Considerando o branco total de $\mathrm{Sr}$ de $2 \mathrm{ng}$, optou-se por analisar cerca de $50 \mathrm{~mL}$ de amostra de água para eliminar um eventual efeito da contaminação laboratorial no procedimento analítico.

\section{APLICAÇÃO DO MÉTODO DE ANÁLISE ISOTÓPICA DE SR EM ÁGUAS NATURAIS}

\section{Área de estudo}

A área selecionada para aplicar a metodologia de análise isotópica de $\mathrm{Sr}$ em águas naturais situa-se no município de Bragança, no nordeste do Estado do Pará, distando 208 km de Belém. Este município pertence à região do Salgado, também denominada de região Bragantina por Ackerman ${ }^{26}$, e está compreendida entre o litoral, ao norte, o vale do rio Guamá, ao sul, o vale do rio Gurupi, ao leste e os rios Pará e Tocantins, a oeste ${ }^{27}$.

O clima da região Bragantina caracteriza-se por ser quente e úmido e do tipo Am 2 (segundo adaptação da classificação de Koppen) destacando-se um período de seca de junho a novembro e um chuvoso bem acentuado, com chuvas fortes de dezembro a maio. O índice pluviométrico médio anual oscila entre 2.500 a $3.000 \mathrm{~mm}^{28}$.

A variação média anual da temperatura para esta região situase entre 21,1 e $30,9{ }^{\circ} \mathrm{C}$, com média de $25,7^{\circ} \mathrm{C}$, atingindo valores máximos de até $42{ }^{\circ} \mathrm{C}$ nos meses de agosto a outubro ${ }^{28}$.

Os locais de coleta de amostra situam-se na Planície Costeira e Planalto Costeiro (Figura 2), que são unidades geomorfológicas da Planície Costeira Bragantina ${ }^{11,29}$.

A Planície Costeira é a mais extensa das unidades morfológicas e é limitada ao sul por falésias mortas de 1 a $2 \mathrm{~m}$ de altura, esculpi-

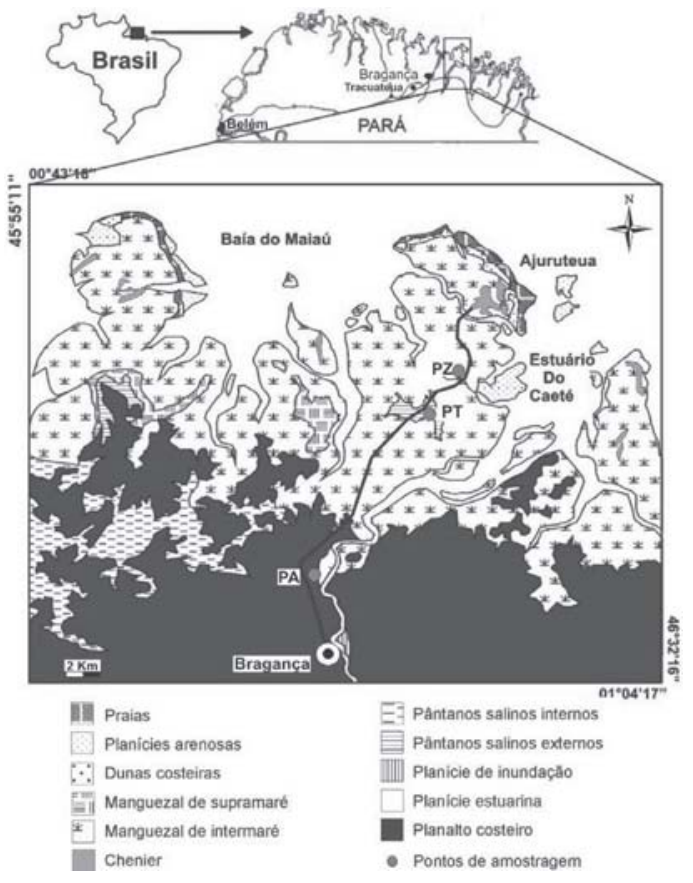

Figura 2. Mapa dos ambientes sedimentares da planície costeira Bragantina mostrando os pontos de amostragem selecionados. Modificado da ref. 11 
das no Planalto Costeiro. Na Planície Costeira são reconhecidos ambientes de pântanos salinos (interno e externo), manguezais (de supra e intermaré), cheniers, planície arenosa, dunas costeiras e praias. O Pântano Salino é representado pelos "Campos de Bragança", que ocupam uma área de $82,7 \mathrm{~km}^{2}$ estando sob influência do domínio da supramaré. Os manguezais são constituídos por sedimentos lamosos, colonizados principalmente por Ryzophora e Avicenia, submetidos à influência das marés, ocupando uma área de $466,8 \mathrm{~km}^{2}$.

O Planalto Costeiro é composto de sedimentos silicáticos do Grupo Barreiras, representa uma superfície plana arrasada, suavemente ondulada e fortemente dissecada, com cotas entre 50 e 60 $\mathrm{m}$, que diminuem progressivamente em direção à Planície Costeira a norte. O contato entre o Planalto Costeiro e a Planície Costeira é marcado por uma mudança litológica (sedimentos areno-argilosos avermelhados do Grupo Barreiras e lamosos da planície costeira), mudança vegetacional brusca (floresta secundária e mangue), e morfológica brusca (falésias mortas de até $1 \mathrm{~m}$ de altura).

\section{Coleta e preparação das amostras}

A amostragem das águas naturais foi realizada em poços ao longo da estrada Bragança-Ajuruteua, nordeste do Estado do Pará (Figura 2). A coleta foi efetuada bimestralmente no período de dezembro de 1998 a outubro de 1999, com objetivo de registrar a variação sazonal da região. Foram coletadas 18 amostras, sendo 3 amostras em cada ida ao campo nos meses de dezembro de 1998, março, maio, julho, agosto e outubro de 1999. Os 3 pontos amostrados foram (PA) poço do tipo amazonas; (PT) poço tubular e (PZ) piezômetro.

O poço do tipo amazonas (PA) situa-se na vila do Acarajó, a 6 $\mathrm{km}$ da cidade de Bragança. Está localizado na região geomorfologicamente definida como Planalto Costeiro, portanto, nos sedimentos silicáticos da Formação Barreiras. Trata-se de um poço escavado de maneira rudimentar, até se atingir o nível freático mais superficial do local. O poço tubular (PT) possui o nível estático variando em torno de $2 \mathrm{~m}$, e foi perfurado no ambiente de Pântano Salino a 22,5 km da cidade de Bragança. O piezômetro (PZ) está localizado em área de mangue intermaré no Furo do Chato, à distância de 29,5 km de Bragança. Estes dois últimos sítios de amostragens localizam-se na região geomorfologicamente definida como Planície Costeira.

Além da coleta para análises isotópicas de $\mathrm{Sr}$, foram realizadas medidas in situ de parâmetros físico-químicos, como temperatura e pH com auxílio do pHmetro portátil, condutividade elétrica, sólidos totais dissolvidos (STD) e salinidade com um condutivímetro portátil. As amostras destinadas à análise isotópica de $\mathrm{Sr}$ foram filtradas em filtros de $0,45 \mu \mathrm{m}$, acidificadas com $\mathrm{HNO}_{3}{ }^{* *}$ até $\mathrm{pH} \sim 2$ e estocadas em garrafas de $500 \mathrm{~mL}$ de polipropileno refrigeradas.

Para determinação da razão isotópica ${ }^{87} \mathrm{Sr} /{ }^{86} \mathrm{Sr}$, cerca de $50 \mathrm{~mL}$ das amostras coletadas nos pontos PA e PT foram evaporados e posteriormente solubilizados com $0,7 \mathrm{~mL}$ de $\mathrm{HNO}_{3} * * 3,5 \mathrm{~mol} / \mathrm{L}$, para separação cromatográfica do $\mathrm{Sr}$ com resina Sr.spec. Para as amostras do ponto $\mathrm{PZ}$, entre $5 \mathrm{e} 10 \mathrm{~mL}$ foram evaporados, devido à alta concentração de sais que resultou na formação de resíduo de sulfato de cálcio. Este resíduo foi dissolvido com $2 \mathrm{~mL} \mathrm{HCl} * * 2,5$ $\mathrm{mol} / \mathrm{L}$, evaporado, em seguida solubilizado com $1,5 \mathrm{~mL}$ de $\mathrm{HNO}_{3} * *$ 3,5 mol/L com auxílio de ultra-som por $3 \mathrm{~min}$, para garantir a completa dissolução da amostra.

A diluição isotópica foi efetuada pela mistura de massas préestabelecidas das amostras e da solução traçadora de Sr.

\section{RESULTADOS ANALÍTICOS}

\section{Parâmetros físico-químicos}

Informações sobre a precipitação pluviométrica da região são importantes para a interpretação dos parâmetros físico-químicos. Em geral, o período entre agosto e novembro é aquele que apresenta menor precipitação (Tabela 1).

No ambiente aquático os processos físicos, químicos e biológicos são afetados pela temperatura. As águas subterrâneas têm temperaturas pouco variáveis que refletem a média anual das temperaturas atmosféricas do local, incrementada pelo gradiente térmico em função da profundidade ${ }^{30}$. Na Tabela 2 observa-se que a temperatura das águas subsuperficias dos pontos de amostragem variou entre 27,1 e $30,8^{\circ} \mathrm{C}$, que é característica do clima da região.

$\mathrm{O}$ pH das águas coletadas nos pontos PA e PT variou de 4,38 a 5,8 , enquanto que no ponto $\mathrm{PZ}$ situou-se sistematicamente em torno de 7 (Tabela 2). Esses valores estão dentro da faixa de $\mathrm{pH}$ das águas naturais, que varia entre 4 e 9 . No ponto PZ, onde a influência marinha é significativa, o valor do $\mathrm{pH}$ aproxima-se mais daquele da água do mar (8 a 8,3).

A condutividade elétrica da água é dependente do seu conteúdo iônico, implicando que uma elevada condutividade elétrica pode indicar influência marinha ${ }^{31}$. Nas águas superficiais, mais comumente, os valores da condutividade elétrica situam-se entre 50 e $1.500 \mu \mathrm{S} / \mathrm{cm}^{32}$, na água do mar entre 45.000 e $55.000 \mu \mathrm{S} / \mathrm{cm}$. As amostras de água do PA forneceram valores de condutividade elétrica entre 46,5 e $89,5 \mu \mathrm{S} / \mathrm{cm}$. Os valores mais baixos foram alcançados nos meses entre dezembro/98 e março/99, onde se registram normalmente os maiores índices pluviométricos da região nordeste do Pará.

Nas amostras de água do PT, a condutividade apresentou-se um pouco mais elevada, variando de 74,9 até $150,5 \mu \mathrm{S} / \mathrm{cm}$ (Tabela 2). No entanto, uma relação entre a sazonalidade e os valores da condutividade não é muito clara, provavelmente, por se tratar de água subsuperficial de um poço tubular, sem contato direto com a precipitação atmosférica.

A condutividade das águas coletadas no $\mathrm{PZ}$ apresentou valores extremamente altos 47.000 e $58.100 \mu \mathrm{S} / \mathrm{cm}$ (Tabela 2). Observa-se ainda neste ponto uma tendência crescente desses valores no sentido dos meses mais secos. Os altos valores observados devem-se ao fato deste piezômetro ser localizado na região de mangue intermaré, onde a água subsuperficial sofre grande influência marinha.

O teor de sólidos totais dissolvidos (STD) é um índice da quantidade de substâncias dissolvidas na água e está diretamente relacionado com a condutividade elétrica. Em águas doces os valores de STD variaram entre 50 e $1500 \mathrm{mg} / \mathrm{L}$ e para a água do mar o

Tabela 1. Total de precipitação mensal (mm) nos anos de 1998 e 1999, coletados na estação meteorológica localizada no município de Tracuateua, NE do Pará, situado a $20 \mathrm{~km}$ da área de estudo (relatório nº14 SEOMA- INMET)

\begin{tabular}{lcccccccccccc}
\hline Anos/Meses & JAN & FEV & MAR & ABR & MAI & JUN & JUL & AGO & SET & OUT & NOV & DEZ \\
\hline 1998 & 458 & 110,3 & 482,5 & 222,1 & 201,3 & 180,7 & 312,2 & 112,3 & 2,2 & 2,4 & 2,4 & $\mathbf{2 1 , 2}$ \\
1999 & 134,7 & 277,4 & $\mathbf{5 6 9 , 0}$ & 294,2 & $\mathbf{4 1 0}$ & 216,4 & $\mathbf{2 3 6 , 4}$ & $\mathbf{1 1 2 , 7}$ & 11,5 & $\mathbf{7 7 , 3}$ & 8,3 & 138,2 \\
\hline
\end{tabular}

Os dados ressaltados em negrito indicam os meses da coleta. 
Tabela 2. Parâmetros físico-químicos das águas subsuperficiais amostradas

\begin{tabular}{|c|c|c|c|c|c|c|c|c|c|c|c|c|c|c|c|c|c|c|c|c|c|c|}
\hline \multirow[b]{2}{*}{ DATA } & \multirow[b]{2}{*}{ LUA } & \multicolumn{7}{|c|}{$\begin{array}{l}\text { Poço amazonas do Acarajó (PA) } \\
01^{\circ} 01^{\prime} 05^{\prime} \text { S e } 046^{\circ} 46^{\prime} 06^{\prime \prime} \mathrm{W}\end{array}$} & \multicolumn{7}{|c|}{$\begin{array}{c}\text { Poço tubular no Pântano Salino (PT) } \\
00^{\circ} 54^{\prime} 42^{\prime \prime} \text { S e } 046^{\circ} 40^{\prime} 49^{\prime \prime} \mathrm{W}\end{array}$} & \multicolumn{7}{|c|}{$\begin{array}{l}\text { Piezômetro no Furo do Chato (PZ) } \\
00^{\circ} 52^{\prime} 14^{\prime \prime} \mathrm{S} \text { e } 046^{\circ} 39^{\prime} 06^{\prime \prime} \mathrm{W}\end{array}$} \\
\hline & & Hora & $\mathrm{pH}$ & $\begin{array}{c}\mathrm{C} \\
\mu \mathrm{S} / \mathrm{cm}\end{array}$ & $\begin{array}{l}\mathrm{T} \\
{ }^{\circ} \mathrm{C}\end{array}$ & $\begin{array}{l}\mathrm{STD} \\
\mathrm{mg} / \mathrm{L}\end{array}$ & $\begin{array}{l}\text { Sal } \\
\% o\end{array}$ & M & Hora & $\mathrm{pH}$ & $\begin{array}{c}\mathrm{C} \\
\mu \mathrm{S} / \mathrm{cm}\end{array}$ & $\begin{array}{c}\mathrm{T} \\
{ }^{\circ} \mathrm{C}\end{array}$ & $\begin{array}{l}\mathrm{STD} \\
\mathrm{mg} / \mathrm{L}\end{array}$ & $\begin{array}{l}\text { Sal } \\
\% o\end{array}$ & M & Hora & $\mathrm{pH}$ & $\begin{array}{c}\mathrm{C} \\
\mu \mathrm{S} / \mathrm{cm}\end{array}$ & $\begin{array}{l}\mathrm{T} \\
{ }^{\circ} \mathrm{C}\end{array}$ & $\begin{array}{l}\mathrm{STD} \\
\mathrm{mg} / \mathrm{L}\end{array}$ & $\begin{array}{l}\text { Sal } \\
\% \circ\end{array}$ & M \\
\hline $15 / 12 / 98$ & $\mathrm{Mg}$ & $11: 30$ & 4,39 & 46,9 & 28,0 & 43 & 0 & $\mathrm{E}$ & $11: 00$ & 4,38 & 150,5 & 30,8 & 132 & 0 & $\mathrm{BM}$ & $9: 20$ & 7,07 & 47000 & 29,9 & - & 30,5 & $\mathrm{~V}$ \\
\hline 09/03/99 & $\mathrm{C}$ & $17: 30$ & 5,80 & 46,5 & 27,1 & 51 & 0 & $\mathrm{BM}$ & $9: 45$ & 5,11 & 74,9 & 29,5 & 81 & 0 & E & $11: 30$ & 7,30 & 50900 & 29,7 & - & 33,4 & V \\
\hline $11 / 05 / 99$ & $\mathrm{Mg}$ & 11:00 & 5,38 & 64,3 & 28,0 & 63 & 0 & $\mathrm{E}$ & $10: 45$ & 5,09 & 86,5 & 28,5 & 85 & 0 & E & $10: 25$ & 7,22 & 56400 & 27,5 & - & 37,5 & $\mathrm{E}$ \\
\hline 08/07/99 & $\mathrm{Mg}$ & 08:40 & 4,64 & 89,5 & 27,8 & 88 & 0 & $\mathrm{E}$ & $10: 20$ & 4,58 & 112,6 & 27,8 & 112 & 0 & $\mathrm{E}$ & $10: 50$ & 7,05 & 55000 & 28,8 & - & 36,5 & $\mathrm{E}$ \\
\hline $31 / 08 / 99$ & $\mathrm{C}$ & $09: 15$ & 4,40 & 67,0 & 28,4 & 63 & 0 & PM & $09: 45$ & 4,26 & 98,0 & 28,9 & 98 & 0 & $\mathrm{PM}$ & $10: 10$ & 6,74 & 58100 & 28,0 & - & 38,8 & PM \\
\hline $25 / 10 / 99$ & $\mathrm{C}$ & $09: 45$ & 5,12 & - & 28,5 & - & - & V & $10: 25$ & 4,42 & - & 30,0 & - & - & V & $11: 15$ & 7,06 & - & 29,4 & - & - & $\mathrm{V}$ \\
\hline
\end{tabular}

Lua; Mg - minguante; C - cheia; M - Maré; E - enchente; BM - baixa-mar; PM - préa-mar; V - vazante; C - condutividade elétrica; T - temperatura; STD - sólidos totais dissolvidos; Sal - salinidade. *Não foi possível o registro dos parâmetros STD, salinidade e condutividade no mês de outubro devido a defeito no equipamento.

conteúdo de STD é em torno de $35.000 \mathrm{mg} / \mathrm{L}^{30}$. Os valores de STD no PA variaram de 43 a $88 \mathrm{mg} / \mathrm{L}$ e nas amostras de água do PT os valores de STD variam entre 81 e $132 \mathrm{mg} / \mathrm{L}$ (Tabela 2). Novamente, não é clara a relação entre este parâmetro e a sazonalidade. Os valores de STD nas águas subsuperficiais do PZ ficaram acima do nível de detecção do equipamento.

A salinidade é levemente menor que o peso total dos sólidos dissolvidos por quilograma, e pode ser expressa em gramas por quilograma $(\mathrm{g} / \mathrm{kg})$ de água do mar, ou em partes por mil (\%o). No oceano aberto, a salinidade média é $35 \% 0^{33}$. Nas águas subsuperficiais amostradas no PA e PT a salinidade é zero ao longo dos meses amostrados, porém nas amostras do PZ a salinidade se apresentou entre 33,4 e 38,8\%o mostrando uma tendência crescente do período chuvoso para o de estiagem (Tabela 2).

\section{Composição isotópica e concentração do $\mathrm{Sr}$}

Os valores da razão isotópica ${ }^{87} \mathrm{Sr} /{ }^{86} \mathrm{Sr}$ são representadas neste trabalho por $\delta^{87} \mathrm{Sr} \%$, calculado em relação à razão ${ }^{87} \mathrm{Sr} /{ }^{86} \mathrm{Sr}$ da água do mar de 0,70920 como proposto por Naftz et al. ${ }^{20}$. Os teores de Sr para as amostras de água coletadas no PA variaram de 13,4 a $58,0 \mu \mathrm{g} / \mathrm{L}$ e os valores de $\delta^{87} \mathrm{Sr}$ entre 1,51 e 6,26\% (Tabela 3). Nos meses de julho, agosto e outubro/99 os valores de $\delta^{87} \mathrm{Sr}$ foram relativamente similares, variando de 3,36 a $3,53 \%$, e a concentração de Sr na água neste período decresceu de 58 para $28,1 \mu \mathrm{g} / \mathrm{L}$. Por outro lado, no mês de maio/99 foi registrado o maior valor de $\delta^{87} \mathrm{Sr}$, $(6,26 \%$ ) e a concentração foi a mais baixa medida $(13,4 \mu \mathrm{g} / \mathrm{L})$. Nos meses de dezembro/98 e março/99, foram registradas concentrações acima de $50 \mu \mathrm{g} / \mathrm{L}$ na água, porém os valores de $\ddot{a}^{87} \mathrm{Sr}$ foram os mais baixos, 1,51 e $1,66 \%$ o respectivamente.

Para as amostras de águas do PT, o valor de $\delta^{87} \mathrm{Sr}$ apresentou-se relativamente uniforme, variando de 0,55 a 090\%, e bem menor que àqueles registrados nas águas do PA. As concentrações de $\mathrm{Sr}$ variaram entre 93,0 e 114,6 $\mu \mathrm{g} / \mathrm{L}$, em geral de 2 a 3 vezes mais elevadas que as amostras do PA (Tabela 3). A concentração de $\mathrm{Sr}$ nas amostras de água do PT foi ligeiramente mais alta nos meses de dezembro/98 e março/99, situando-se em torno de $114 \mu \mathrm{g} / \mathrm{L}$, nos demais meses esta concentração situou-se entre 93 e $104 \mu \mathrm{g} / \mathrm{L}$. Por outro lado, os meses de dezembro/98 e março/99 apresentaram valores mais baixos de $\delta^{87} \mathrm{Sr}$, respectivamente, 0,55 e $0,76 \%$, enquanto que nos meses subseqüentes este valor foi levemente mais alto, variando de 0,79 até $0,90 \%$.

Na Tabela 3 observa-se ainda que, as amostras de água do PZ, localizado no Furo do Chato, teve valores de $\delta^{87} \mathrm{Sr}$ variando de $-0,18$ a $0,18 \%$ e as concentrações de $\mathrm{Sr}$ extremamente elevadas, situandose entre 15,44 e 23,25 mg/L com um valor extremo de 67,7 mg/L, no mês de março/99. Estes valores bem mais elevados de Sr neste ambiente eram esperados em função destas águas possuírem alta salinidade, refletindo uma contribuição direta da água do mar.

\section{DISCUSSÃO}

A composição isotópica do $\mathrm{Sr}$ reflete claramente os diferentes ambientes onde as águas subsuperficiais foram amostradas. No piezômetro do Furo do Chato, situado no manguezal de intermaré onde a influência marinha é evidente, os valores da razão ${ }^{87} \mathrm{Sr} /{ }^{86} \mathrm{Sr}$ são similares à razão isotópica da água do $\operatorname{mar}^{16} \mathrm{e}$ os valores de $\delta^{87} \mathrm{Sr}$ muito próximos de zero. Os valores de $\delta^{87} \mathrm{Sr}$ observados neste poço nos meses de julho e agosto/99, que se afastam desta tendência, podem representar pequenas variações locais da composição isotópica da água do mar. Os parâmetros físico-químicos medidos (condutividade, salinidade e $\mathrm{pH}$ ), o teor de $\mathrm{Sr}$ e a razão ${ }^{87} \mathrm{Sr} /{ }^{86} \mathrm{Sr}$

Tabela 3. Concentração de $\mathrm{Sr}$, razão isotópica ${ }^{87} \mathrm{Sr} /{ }^{86} \mathrm{Sr}$ e $\delta^{87} \mathrm{Sr}(\%$ )* nas águas subsuperficiais amostradas

\begin{tabular}{|c|c|c|c|c|c|c|c|c|c|}
\hline \multirow[b]{2}{*}{ DATA } & \multicolumn{3}{|c|}{ Poço Amazonas Acarajó (PA) } & \multicolumn{3}{|c|}{ Poço Tubular Pântano Salino (PT) } & \multicolumn{3}{|c|}{ Piezômetro no Furo do Chato (PZ) } \\
\hline & $\begin{array}{l}\text { CSr total } \\
(\mu \mathrm{g} / \mathrm{L})\end{array}$ & $\left({ }^{87} \mathrm{Sr} /{ }^{86} \mathrm{Sr}\right)$ & $\begin{array}{l}\delta^{87} \mathrm{Sr} \\
(\% \circ)\end{array}$ & $\begin{array}{l}\text { CSr total } \\
(\mu \mathrm{g} / \mathrm{L})\end{array}$ & $\left({ }^{87} \mathrm{Sr} /{ }^{86} \mathrm{Sr}\right)$ & $\begin{array}{l}\delta^{87} \mathrm{Sr} \\
(\% 0)\end{array}$ & $\begin{array}{l}\text { CSr total } \\
(\mathrm{mg} / \mathrm{L})\end{array}$ & $\left({ }^{87} \mathrm{Sr} /{ }^{86} \mathrm{Sr}\right)$ & $\begin{array}{l}\delta^{87} \mathrm{Sr} \\
(\% 0)\end{array}$ \\
\hline Dez/98 & 57,8 & $0,71027 \pm 0,00008$ & 1,51 & 113,6 & $0,70959 \pm 0,00006$ & 0,55 & 15,4 & $0,70914 \pm 0,00004$ & $-0,08$ \\
\hline Mar/99 & 4,9 & $71038 \pm 0,00008$ & 1,6 & 114,7 & $0,70974 \pm 0,00006$ & 0,7 & 67,7 & $0,70921 \pm 0,00007$ & 0,01 \\
\hline Mai/99 & 13,4 & $0,71364 \pm 0,00009$ & 6,26 & 98,1 & $0,70979 \pm 0,00003$ & 0,83 & 20,2 & $0,70916 \pm 0,00006$ & $-0,06$ \\
\hline Jul/99 & 58,0 & $0,71170 \pm 0,00006$ & 3,53 & 103,6 & $0,70977 \pm 0,00002$ & 0,80 & 23,2 & $0,70933 \pm 0,00006$ & 0,18 \\
\hline Ago/99 & 32,0 & $0,71161 \pm 0,00004$ & 3,40 & 93,0 & $0,70976 \pm 0,00005$ & 0,79 & 22,2 & $0,70907 \pm 0,00006$ & $-0,18$ \\
\hline Out/99 & 28,1 & $0,71158 \pm 0,00004$ & 3,36 & 97,2 & $0,70984 \pm 0,00006$ & 0,90 & 21,6 & $0,70917 \pm 0,00005$ & $-0,04$ \\
\hline
\end{tabular}

$* \delta^{87} \mathrm{Sr}=\left\{\left[\left({ }^{87} \mathrm{Sr} /{ }^{86} \mathrm{Sr}\right) \mathrm{m} /\left({ }^{87} \mathrm{Sr} /{ }^{86} \mathrm{Sr}\right) \mathrm{sw}\right]-1\right\} \times 1000{ }^{20} ;\left({ }^{87} \mathrm{Sr} /{ }^{86} \mathrm{Sr}\right) \mathrm{m}=$ razão isotópica medida; $\left({ }^{87} \mathrm{Sr} /{ }^{86} \mathrm{Sr}\right) \mathrm{sw}=0,70920$, valor da razão ${ }^{87} \mathrm{Sr} /{ }^{86} \mathrm{Sr}$ na água do $\mathrm{mar}^{20}$ 
indicam claramente a contribuição das águas marinhas nessas águas subsuperficias (Tabelas 2 e 3 ).

A elevada concentração de $\mathrm{Sr}$ das águas deste poço, muito superior àquela da água do mar, situada em torno de $8 \mathrm{mg} / \mathrm{L}^{12}$, deve se provavelmente à evaporação mais acelerada nessa zona de manguezal intermaré. O valor anômalo de $67,7 \mathrm{mg} / \mathrm{L}$ de $\mathrm{Sr}$ observado em março/99 pode estar associado à ocorrência de maré de sizígia, ou seja, épocas de grandes marés no equinócio em lua cheia. Nessa ocasião, ocorre o avanço significativo da água do mar na região de manguezal, provocando a remobilização dos sedimentos inconsolidados e o aumento de sólidos dissolvidos na água.

No outro extremo tem-se o poço amazonas da Vila do Acarajó, escavado em sedimentos continentais e diretamente influenciado por águas pluviais. A razão ${ }^{87} \mathrm{Sr} /{ }^{66} \mathrm{Sr}$ possui uma assinatura isotópica típica de águas subsuperficias continentais, e os valores de $\delta^{87} \mathrm{Sr}$ podem ser agrupados em três conjuntos distintos (Tabela 3). Nos meses de dezembro/98 e março/99 encontraram-se valores mais baixos de $\delta^{87} \mathrm{Sr}(1,51-1,61 \%$ ). Em maio/99, tem-se o mais alto valor de $\delta^{87} \mathrm{Sr}(6,26 \%$ ), enquanto que nos meses de julho a outubro de 1999 observam-se valores intermediários de $\delta^{87} \mathrm{Sr}$ (3,36-3,53\%o). Acredita-se que o valor de $\delta^{87} \mathrm{Sr}$ obtido em maio/99, nas águas do poço $\mathrm{PA}$, represente mais fielmente a composição isotópica média das rochas continentais da região, ou seja, maior contribuição de $\mathrm{Sr}$ radiogênico proveniente das rochas percoladas pelas águas de subsuperfície que fluem para esse poço. Além disso, a mais baixa concentração de $\mathrm{Sr}(13 \mu \mathrm{g} / \mathrm{L})$ pode estar refletindo a diluição deste elemento como resultado do efeito retardado do índice pluviométrico mais alto do período.

O poço tubular, situado no Pântano Salino, estaria na situação intermediária entre os dois ambientes anteriormente discutidos, com assinatura isotópica próxima da água do mar, porém com uma pequena contribuição de $\mathrm{Sr}$ radiogênico. O intervalo da concentração de $\mathrm{Sr}(93-115 \mu \mathrm{g} / \mathrm{L})$ dessas águas subsuperficiais é bem inferior ao teor encontrado em águas marinhas e os parâmetros físico-químicos guardam maior semelhança com aqueles medidos no poço amazonas que no Furo do Chato (Tabela 2). Estes dados sugerem, portanto, uma influência muito limitada de águas marinhas. Por outro lado, os valores de $\delta^{87} \mathrm{Sr}$ situados entre $0,55-0,90 \%$, mostram que a composição isotópica é muito próxima daquela da água do mar e distinta das águas continentais do poço amazonas. Acredita-se que os valores de $\delta^{87} \mathrm{Sr}$ reflitam o fato dessas águas estarem percolando sedimentos formados em ambiente transicional com influência marinha durante a sua deposição.

\section{CONCLUSÃO}

A determinação das razões isotópicas ${ }^{87} \mathrm{Sr} /{ }^{86} \mathrm{Sr}$ e das concentrações de $\mathrm{Sr}$ nas amostras de águas subsuperficiais coletadas na região Bragantina mostram que a utilização de resina específica de Sr (Sr.spec) para separação cromatográfica deste elemento, é adequada para amostras de águas naturais. Isto se deve principalmente às separações químicas obtidas com elevada resolução que levaram à eliminação do cálcio e do rubídio, principais interferentes na análise isotópica do estrôncio.

Outra vantagem desse método é a utilização de apenas dois eluentes $\left(\mathrm{H}_{2} \mathrm{O}\right.$ e $\left.\mathrm{HNO}_{3}\right)$, o que torna esse procedimento simples e menos exaustivo que aqueles empregados em outras técnicas de separação cromatográfica de Sr. A utilização de uma reduzida quantidade de reagentes, a limpeza rigorosa do laboratório e dos materiais utilizados, bem como a purificação dos reagentes, são decisivos para a redução do branco total de Sr, possibilitando a análise de materiais com baixa concentração de Sr. Portanto, o emprego desse método, além de tornar possível a utilização da composição isotópica de $\mathrm{Sr}$ em estudos ambientais, permite reduzir a quantidade de amostra necessária para estudos isotópicos.

A aplicação desta técnica em águas subsuperficiais na zona costeira da região Bragantina, nordeste do Estado do Pará, mostrou que essas águas possuem composições isotópicas distintas e refletem a composição isotópica das rochas ou sedimentos que elas percolaram.

Nos sedimentos continentais da região do Acarajó os valores de $\delta^{87} \mathrm{Sr}(1,51-6,26 \%$ o ) foram mais altos, refletindo maior contribuição de Sr radiogênico compatível com o ambiente continental. Acreditase que o valor de $\delta^{87} \mathrm{Sr}$ de $6,26 \%$ seja o mais próximo da assinatura isotópica média do $\mathrm{Sr}$ das rochas continentais terciárias desta região. Contrariamente, no piezômetro Furo do Chato, localizado em ambiente de mangue intermaré, a influência marinha nas águas subsuperficiais está claramente retratada nos valores de $\delta^{87} \mathrm{Sr}$ próximos de zero, na alta concentração de $\mathrm{Sr}$ e na salinidade e condutividade destas águas. Nas amostras de água subsuperficias do poço tubular no Pântano Salino, os valores de $\delta^{87} \mathrm{Sr}(0,55-0,90 \%$ ) registram que essas águas percolam sedimentos formados em ambiente transicional com influência marinha durante a sua deposição. No entanto, esta influência é limitada, uma vez que a salinidade, a condutividade e a concentração de $\mathrm{Sr}$ são mais compatíveis com as águas subsuperficiais que percolam rochas continentais.

\section{AGRADECIMENTOS}

Ao apoio do Projeto Madam (Dinamic and Management of Mangrove) e do Curso de Pós-Graduação em Geologia- CPGG. Durante a realização deste trabalho o primeiro autor recebeu bolsa do Conselho Nacional de Desenvolvimento Científico e Tecnológico (CNPQ). As análises foram realizadas no Laboratório de Geologia Isotópica da Universidade Federal do Pará - Pará-Iso, com o apoio técnico de C. M. Silva.

\section{REFERÊNCIAS}

1. Palmer, M. R.; Edmond, J. M.; Geochim. Cosmochim. Acta 1992, 56, 2099.

2. Lyons, W. B.; Tyler, S. W.; Gaudette, H. E.; Long, D. T.; J. Hydrol. 1995, 187, 225.

3. Allégre, C. J.; Dupré, B.; Négrel, P.; Gaillardet, J.; Chem. Geol. 1996, 131, 93.

4. Bullen, T. D.; Krabbenhoft, D. P.; Kendall, C.; Geochim Cosmochim. Acta 1996, 60, 1807.

5. Siegel, D. I.; Bickford, M. E.; Orrel, S. E.; Appl. Geochem. 2000, 15, 493.

6. Charalampides, G.; Manoliades, O.; Environment International 2002, 28, 147.

7. Bohlke, J. K.; Horan, M.; Appl. Geochem. 2000, 15, 599.

8. Gosselin, D. C.; Harvey, F. E.; Frost, C.; Stotelr, R.; Macfarlane, P. A.; Appl. Geochem. 2004, 19, 359.

9. Kurkjian, R.; Dunlap, C.; Flegal, A. R.; Appl Geochem. 2004, 19, 1567.

10. Horwitz, E. P.; Dietz, M. L.; Chiarizia, R.; Solvent Extr. Ion Exch. 1992, 10,313 .

11. Souza Filho, P. W. M.; El Robrini, M.; Bol. Mus. Para. Emílio Goeldi 1998, $10,45$.

12. Faure, G.; Principles of Isotope Geology, $2^{\text {nd }}$ ed.; John Wiley \& Sons: New York, 1986.

13. Banner, J. L.; Earth Science Reviews 2004, 65, 141.

14. Banner, J. L.; Musgrove, M.; Capo, R. C.; Geology 1994, 22, 687.

15. Herut, B.; Starinsky, A.; Katz, A.; Earth Planet. Sci. Lett. 1993, 120, 77.

16. Kawashita, K.; Marques, F.; Soares, E.; Pinto, M. S.; Actas 10 ${ }^{a}$ Semana de Geoquímica/Iv Congresso de Geoquímica dos Países de Língua Portuguesa, Braga, Portugal, 1997.

17. Thomaz Filho, A.; Mizusaki, A. M. P.; Kawashita, K.; Torquato, J. R.; Revista de Geologia 1995, 8, 213.

18. Burke, W. H.; Denison, R. E.; Hetherington, E. A.; Koepnick, R. B.; Nelson, H. F.; Otto, J. B.; Geology 1982, 10, 516.

19. Collerson, K. D.; Ullman,W. J.; Jorgersen, T.; Geology 1988, 16, 59.

20. Naftz, D. L.; Peterman, Z. E.; Spangler, L. E.; Chem. Geol. 1997, 141, 195.

21. Horwitz, E. P.; Dietz, M. L.; Fisher, E. D.; Anal. Chem. 1991, 63, 522. 
22. Pin, C.; Bassin, C.; Anal. Chim. Acta 1992, 269, 249.

23. Horwitz, E. P.; Dietz, M. L.; Fisher, E. D.; Solvent Extr. Ion Exch. 1990 8 , issues 4 e 5 .

24. Moore, L. J.; Moody, J. R.; Barnes, I. L.; Gramlich, J. W.; Murphy, T. J.; Paulsen, P. J.; Shields, W. R.; Anal Chem. 1973, 45, 2384.

25. Henderson, P.; Rare Earth Element Geochemistry, Elsevier: New York, 1984.

26. Ackerman, F. L.; Cadernos da Amazônia 1964, 2, 92.

27. Francisco,B. H. R.; Loewenstein, P.; Silva, O. F.; Silva, G. G.; Bol. Mus. Para. Emílio Goeldi 1971, 17, 1.
28. Martorano, L. G.; Perreira, L. C.; Cézar, E. G. M.; Pereira, I. C. B.; Estudos Climáticos do Estado do Pará, Classificação Climática (Koppen) e Deficiência Hídrica (Thornthwhite, Mather), Sudam/Embrapa: Belém, 1993.

29. Souza Filho, P. W. M.; El-Robrini, M.; Revista Brasileira de Geociências 2000, 30, 518 .

30. Custodio, E.; Llamas, M. R.; Hidrologia Subterrânea, $1^{\mathrm{a} e d ., ~ E d . ~ O m e g a: ~}$ Barcelona, 1976.

31. Hem, J. D.; U. S. Geol. Surv. Water-Supply Paper 1970, 1473.

32. Bittencourt, A. V. L.; Hidroquímica, $1^{\circ}$ Curso Sulamericano sobre Avaliação e Vulnerabilidade de Aquíferos, Departamento de Geologia, UFPR, 1994.

33. Mason, B. H.; Princípios de Geoquímica, Ed. USP: São Paulo, 1971. 